\title{
loi expérimentale de propagation des vibrations dues aux tirs d'explosifs
}

\author{
par \\ P. Chapot \\ Laboratoire Régional des Ponts et Chaussées, Nancy
}

RESUME - Des mesures de vibrations provoquées par des tirs d'explosifs au cours de chantiers de travaux publics ont été analysées dans le but de mettre en évidence une relation utilisable pour apprécier a priori les difficultés susceptibles d'etre rencontrées lors de l'exécution de travaux à l'explosif.

\section{INTRODUCTION}

L'exécution de travaux à l'explosif en site construit pose le problème des dangers présentés par les vibrations transmises par les tirs aux constructions.

S'il est actuellement possible de mener à bien de tels travaux en pratiquant un contrôle des vibrations provoquées, il est souvent souhaitable de connaître avant le début des chantiers le niveau probable qui sera atteint par les vibrations.

Il est en effet particulièremnet important de savoir, pour l'estimation du coat des travaux, si les charges à mettre en oeuvre devront être limitées, et si possible à quelle valeur. Ceci intéresse autant le maitre d'oeuvre que l'entreprise établissant un devis. Plus simplement, il peut être nécessaire de savoir si les travaux doivent faire l'objet d'une surveillance ou s'ils peuvent se dérouler sans contrainte.

Les mesures de vibration réalisées au cours de divers chantiers de déroctage à I'explosif ont été utilisées pour tenter de trouver une relation permettant d'établir de telles prévisions.

\section{CHOIX DE IA MESURE}

Les mesures utilisées sont des mesures de vitesse de vibration, effectuées à l'aide de géophones tridirectionnels dont on retient la valeur maximum.

On peut retenir comme élément significatif :

la valeur mesurée selon la verticale

la plus grande des 3 valeurs mesurées

le maximum de la vitesse résultante.

Les corrélations ont été tentées, chaque fois que cela a été possible, sur ces trois valeurs. Bien qu'il n'y ait pas une trés grande différence entre les corrélations obtenues, c'est incontestablement la vitesse résultante qui donne les meilleurs résultats, suivie par la vitesse selon la verticale. Ia vitesse maximum dans une direction quelconque est un peu plus dispersée. Cependant, la vitesse maximum étant plus proche de la vitesse résultante, $c^{\prime}$ est celle-ci qui a été retenue lorsque le calcul de la vitesse résultante n'était pas possible, ce qui a pu être le cas lorsque la forme des signaux était trop complexe, ou les fréquences trop élevées.

\section{CHOIX DES PARAMETRES}

Nous n'avons pas cherché à prendre en compte tous les éléments susceptibles d'intervenir dans la détermination du niveau de vibration atteint. Ies seuls éléments facilement mesurables sont la quantité d'explosif utilisée, et la distance entre le point de mesure et la zone du tir. D'autres éléments sont certainement importants mais plus difficiles à prendre en compte. On peut citer la nature géologique du terrain et sa structure, la nature de l'explosif, les caractéristiques du plan de tir (géométrie de la maille, séquence de mise à feu).

Nous avons donc cherché des modẽles ne tenant compte explicitement que de la charge et de la distance, les autres paramètres étant pris en compte globalement et supposés constants pour un site donné, c'est à dire pratiquement pour un chantier donné, pour autant que la géologie et la technique utilisée restent semblables.

Les analyses portent sur plus de 600 valeurs effectuées sur 15 sites différents. Environ 350 proviennent de terrassements classiques sur des chantiers de travaux publics au cours desquels le niveau des vibrations produites sur les constructions environnantes a été contrôlé. 
Les valeurs utilisées comprennent donc des mesures effectuées au sol mais aussi sur des immeubles (à la base de ceux-ci) ce qui explique en partie la dispersion des mesures

\section{INFLUENCE DE IA DISTANCE}

L'effet de la distance est relativement facile à mettre en évidence en effectuant des mesures à des distances variées du point de tir au cours d'un tir.

Le report sur un diagrame en coordonnées bilogarithmiques des vitesses maximum en fonction de la distance $D$ au point de tir donne généralement une droite, ce qui exprime une relation de la forme :

$$
\mathrm{v}=\mathrm{KD}^{-\mathrm{b}}
$$

d étant la pente de la droite expérimentale.

Ies valeurs obtenues pour $\mathrm{b}$ sont variables mais toujours comprises entre 1 et 2,5 et le plus souvent entre 1,5 et 2 .

Certains auteurs ont proposé des modèles faisant intervenir un terme d'amortissement exponentiel.

D'autre part la théorie implique que selon le type d'onde, 1'atténuation géométrique peut être proportionnelle à

$$
\frac{I}{D}, \frac{I}{\sqrt{D}} \text { ou } \frac{I}{D^{2}}
$$

Une atténuation conme $\frac{I}{D^{2}} \quad n^{\prime}$ est pas à prendre en considération puisque l'exposant b est le plus souvent inférieur à 2 .

Nous avons donc systématiquement appliqué à nos mesures les modèles suivants, dans lesquels $\mathrm{K}$ représente une constante qui dépend du site étudié

$$
\begin{aligned}
& \mathrm{v}=K \mathrm{D}^{-\mathrm{b}} \\
& \mathrm{v}=\mathrm{K} \frac{\mathrm{I}}{\mathrm{D} n} \mathrm{e}^{-\alpha \mathrm{D}} \\
& \mathrm{v}=\mathrm{K} \frac{1}{\mathrm{D}} \mathrm{e}^{-\alpha \mathrm{D}} \\
& \mathrm{v}=\mathrm{K} \frac{1}{\sqrt{D}} \mathrm{e}^{-\alpha \mathrm{D}}
\end{aligned}
$$

Pour éliminer l'effet de la charge Q, nous avons admis que celle-ci intervenait corme $\Omega 0,75$, ce qui sera justifié plus loin.

Ces mesures permettent les conclusions suivantes :

- la vitesse résultante permet les meilleures corrélations entre vitesse calculée et vitesse mesurée, quelque soit la relation adoptée.

- à défaut de la vitesse résultante, c'est la vitesse verticale (ou la vitesse horizontale transverse dans le cas des mesures en tunnel) qui donne la meilleure corrélation. La plus grande des trois composantes montre une plus grande dispersion.
- la relation 2, avec détermination simultanée de $r$ et $\alpha$ se révèle peu commode : le coefficient du terme exponentiel n'est pas toujours significativement différent de zêro, et la valeur obtenue est parfois positive, ne traduisant donc pas le terme d'amortissement recherché.

- les relations 3 et 4 donnent des résultats beaucoup plus cohérents, la relation (3) étant meilleure que la relation (4) (11 fois sur 12 pour les tirs de surface)

- I'adjonction d'un terme d'amortissement exponentiel n'améliore qu'assez peu la corrélation : quelques unités pour cent sur le coefficient, et ne semble donc pas justifier la complication du calcul dans le cas des mesures de chantier. Ceci n'implique pas qu'une telle relation constitue un modèle inadapté, mais plutôt que la dispersion des mesures utilisées ne permet pas un ajustement suffisamment précis.

\section{INFLUENCE DE LA CHARGE}

On sait qu'en ce qui concerne les vibrations, c'est la charge par unité de mise à feu qui est déterminante, c'est à dire les charges élémentaires attelées à des détonateurs de même micro-retand, ou mises à feu nar un cordeau détonant

(Dans les détonateurs à retard ordinaires, la dispersion entre les détonateurs d'un même numéro est telle que l'on ne peut parler de véritable simultanéîté d'explosion sans condeau détonant).

Dans ce qui suit nous ne parlerons donc que des charges instantannées (désignées par Q) et non pas de la charge totale d'une volée. La presque totalité des mesures utilisées ayant été effectuée sur des chantiers c'est d'ailleurs ce type de charge qui a été effectivement pris en compte.

Dans les calculs théoriques simulant une explosion, une charge est prise en compte comme une pression à l'origine régnant dans une cavité initiale caractérisée par ses dimensions géométriques. Dans une symétrie sphérique, le rayon de cette cavité est supposé proportionnel au volume rééllement occupé par I'explosif, donc si l'explosif est caractérisé par son poids, ce rayon est proportionnel à $Q^{1 / 3}$. Dans une symétrie cylindrique, si l'on modifie la quantité d'explosif par variation du diamètre, le rayon de la cavité initiale varie coume $Q^{1 / 2}$. Dans les tirs rééls de chantier, les charges sont rarement isolées, et les tirs sont constitués d'une volée plus ou moins étendue où il est difficile de replacer les schémas théoriques.

D'un point de vue expérimental, il est possible de procéder à des mesures à charges variables, tous les autres paramètres restant constants, (en particulier la distance) à cette réserve près qu'il n'est pas possible de refaire deux tirs dans des conditions rééllement identiques.

On peut alors rechercher une relation exprimant la 
vitesse maximum de la forme:

$$
\mathrm{v}=\mathrm{K} \Omega^{\mathrm{d}}
$$

Les paramètres $\mathrm{K}$ et a peuvent alors être déterminés par une régression bilogarithmique.

Sur trois sites différents on a ainsi obtenu les résultats suivants :

\section{Site $n^{\circ} 1$}

Mesure de la vitesse verticale au cours de tirs expérimentaux en forage. Charge de 1 à $15 \mathrm{~kg}$.

$$
\mathrm{a}=0,68 \text { (moyenne de } 4 \text { capteurs) }
$$

Site $n^{\circ} 2$

Chantier autoroutier - charge de 6 à $50 \mathrm{~kg}$, (4 tirs, 3 capteurs)

$$
a=0,65
$$

Site $n^{\circ} 3$

Petit abattage d'essai, charges de 0,1 à $0,5 \mathrm{~kg}$

$$
\mathrm{a}=0,63 \text { à } 0,71 \text { selon le capteur. }
$$

La figure 1 montre les résultats relatifs au site $n^{\circ} 2$.

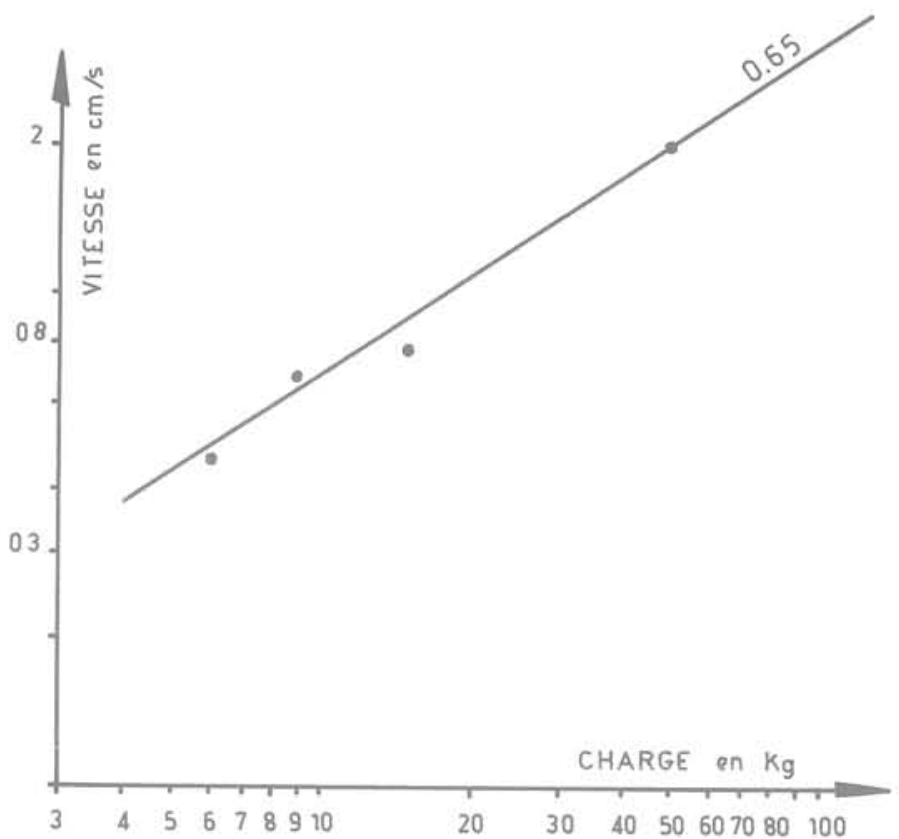

figure 1 - Influence de la charge (site $\mathrm{n}^{\circ} 2$, moyenne de 3 capteurs)

Ces valeurs sont conformes à celles citées par d'autres auteurs, comprises entre 0,5 et 0,9 .

Les mesures effectuées à distance constante avec des charges variant dans un intervalle assez grand étant peu fréquentes sur les chantiers, nous avons utilisé pour la détermination de l'exposant a toutes les mesures disponibles en effectuant une régression portant simultanément sur la distance et la charge selon le modèle:

$$
\mathrm{v}=\mathrm{K} \mathrm{Q}^{\mathrm{a}} \mathrm{D}^{-\mathrm{b}}
$$

soit

$$
\log v=\log K+a \log \Omega-b \log D
$$

En ne retenant que les séries de mesure pour lesquelles le coefficient de corrélation partielle entre $\log v$ et $\log \Omega$ est supérieur à 0,7 on parvient aux résultats du tableau 1.

On peut constater sur ce tableau que la partie commune aux différents intervalles de confiance sur a se situe vers 0,75 . C'est pourquoi cette valeur a été retenue pour éliminer l'influence de la charge dans la recherche de l'influence de la distance.

Tableau 1 - Influence de la charge

\begin{tabular}{|c|c|c|c|c|}
\hline Site & $\begin{array}{c}\text { Nombre } \\
\text { de } \\
\text { valeurs }\end{array}$ & $\begin{array}{c}\text { coefficient } \\
\text { de } \\
\text { corrélation }\end{array}$ & $\begin{array}{c}\text { Valeur } \\
\text { de a }\end{array}$ & $\begin{array}{c}\text { intervalle } \\
\text { de } \\
\text { confiance } \\
\text { à } 0,95\end{array}$ \\
\hline 1 & $2 \overline{1}$ & $-0, \overline{85}$ & $-\overline{0,70}$ & $0, \overline{5} \overline{1}-0, \overline{89}$ \\
2 & 13 & 0,95 & 0,86 & $0,66-1,06$ \\
3 & 63 & 0,83 & 0,86 & $0,72-1,01$ \\
4 & 76 & 0,71 & 0,75 & $0,58-0,91$ \\
5 & 27 & 0,86 & 0,78 & $0,59-0,97$ \\
6 & 17 & 0,70 & 0,70 & $0,34-1,28$ \\
\hline
\end{tabular}

\section{REDUCTION DE LA DISTANCE PAR LA CHARGE}

Toutes les relations qui précèdent expriment la vitesse maximum de vibration en fonction de deux paramètres, charge et distance. De nombreux auteurs ont cherché à se ramener à un seul en réduisant la distance soit par la racine cubique de la charge, soit par la racine carrée.

Le choix entre les deux procédés dépend des considérations théoriques auxquelles on se réfère.

Nous avons systématiquement appliqué à nos mesures les modèles :

$$
\begin{aligned}
& v=k\left(\frac{D}{\sqrt{n}}\right)^{-b} \\
& v=k\left(\frac{D}{\sqrt{\Omega}}\right)^{-b}
\end{aligned}
$$

et

Les ajustements obtenus ne sont pas trés différents mais cependant légèrement meilleurs avec la réduction par la racine carrée. L'exposant $b$ est un peu plus élevé avec la racine cubique (proche de 1,9 au lieu de 1,8 pour la racine carrée). 


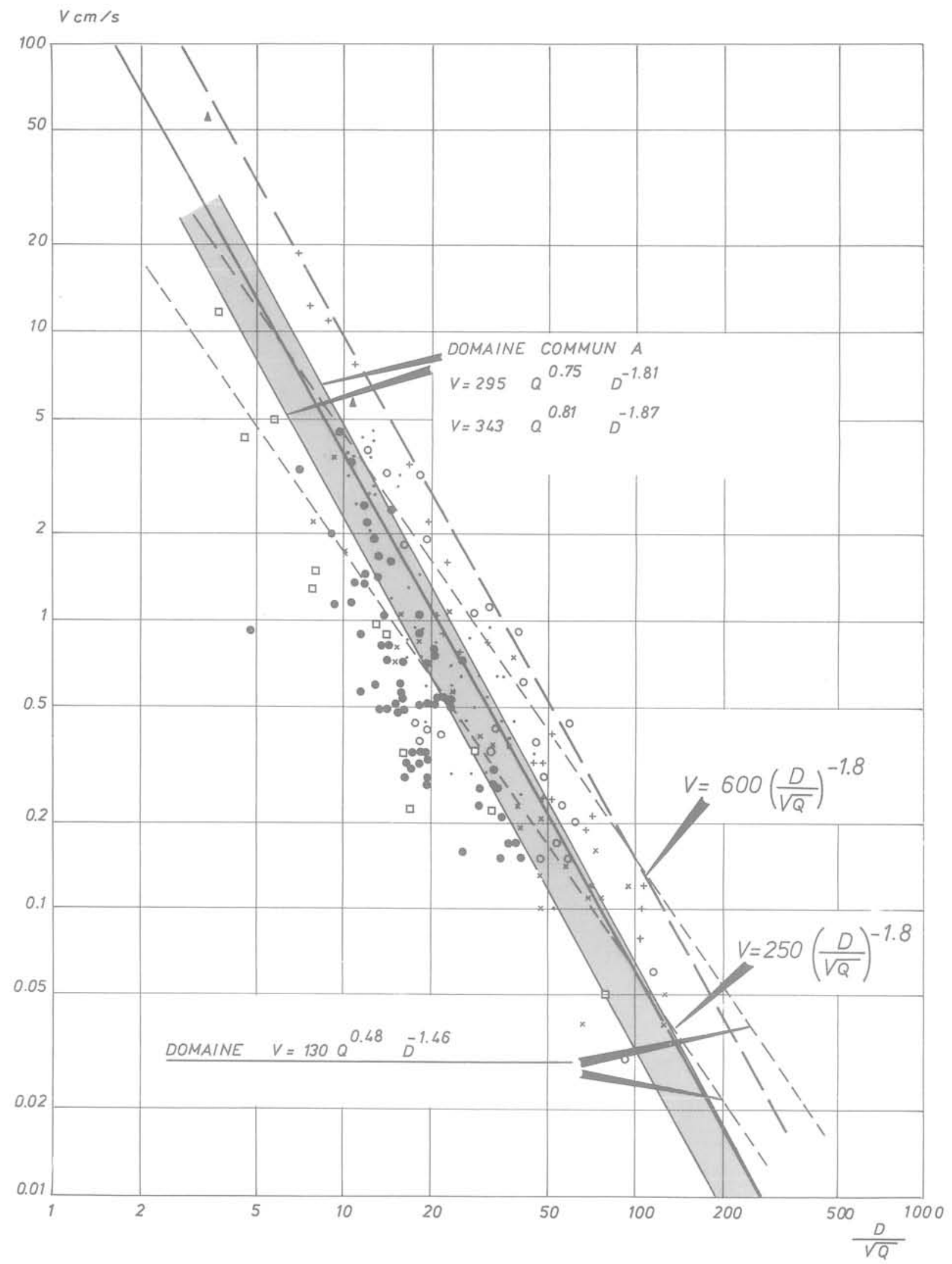

Figure 2 - Vitesse maximum de vibration en fonction de la distance réduite par la racine carrée de la charge. 
RECHERCHE D'UN MODEIE GENERAL

Nous avons vu que trois modèles donnaient des ajustements également satisfaisants :

$$
\begin{aligned}
& \mathrm{V}=K Q^{0,75} \mathrm{D}^{-\mathrm{b}} \\
& \mathrm{V}=\mathrm{K} \mathrm{Q}^{\mathrm{a}} \mathrm{D}^{-\mathrm{b}} \\
& \mathrm{V}=\mathrm{K}\left(\frac{\mathrm{D}}{\sqrt{\Omega}}\right)
\end{aligned}
$$

En retenant les valeurs moyennes des paramètres $K$, $\mathrm{a}$ et $\mathrm{b}$ on obtient respectivement :

$$
\begin{aligned}
& \mathrm{V}=295 \mathrm{Q}^{0,75} \mathrm{D}^{-1,81} \\
& \mathrm{~V}=343 \mathrm{Q}^{0,81} \mathrm{D}^{-1,87} \\
& \mathrm{~V}=250\left(\frac{\mathrm{D}}{\mathrm{Q}}\right)-1,79
\end{aligned}
$$$$
\text { soit } \quad V=250 Q^{0,895} \mathrm{D}^{-1,79}
$$

Ces trois relations sont assez voisines et les variations dans les divers coefficients reflètent la dispersion des mesures, l'influence de la distance étant généralement prépondérante sur celie de la charge, du fait de son domaine de variation généralement plus étendu dans le cas des mesures faites sur chantiers.

La relation utilisant le paramètre $\mathrm{D} / \sqrt{\mathrm{Q}}$ se révèlant la plus commode pour une représentation graphique, nous 1 'avons utilisée dans la figure 2 où sont reportés des résultats expérimentaux.

Nous avons calculé pour les deux autres relations les vitesses en fonction du paramètre $\mathrm{D} / \sqrt{\mathrm{Q}}$, pour des charges de 1 à $50 \mathrm{~kg}$, et des distances de 10 à $1000 \mathrm{~m}$, variables selon la charge de façon à rester dans un domaine de vitesse compris entre 0,01 et $20 \mathrm{~cm} / \mathrm{s}$ ).

Le domaine où sont vérifiées ces relations a été porté sur la même figure : on peut constater que les écarts sont assez faibles, compte tenu de la dispersion habituelle des mesures.

On doit noter que pour les divers paramètres on a retenu la moyenne des valeurs obtenues sur chaque site, alors que la constante $\mathrm{K}$ en particulier est réputée spécifique de chaque site : sa valeur moyenne n'est donc qu'une valeur parmi d'autres possibles. Les valeurs trouvées pour les exposants $\mathrm{a}$ et $\mathrm{b}$ sont par contre suffisamment proches pour être incluses dans un même intervalle de confiance et être par conséquent considérées comme correspondant à une valeur unique avec une probabilité assez élevée.

Nous avons également porté sur la figure 2 le domaine correspondant, pour les mêmes valeurs de charge, à la relation :

$$
V=130 Q^{0,4 \theta} D^{-1,46}
$$

qui est celle proposée par Hendron et Dowding à partir de données expérimentales en utilisant une réduction de la distance par la racine cubique de la charge. S'il existe un domaine commun assez éten- du vers les vitesses moyennes, l'ensemble de nos points expérimentaux en particulier vers les vitesses élevées, semble s'écarter de cette relation.

Si l'on s'attache aux valeurs maximum observées, on constate sur la figure 2 que 1 'on peut tracer une droite parallèle à la droite myenne qui constitue l'enveloppe des points expérimentaux, et qui représente la relation :

$$
v<600\left(\frac{D}{\sqrt{Q}}\right)^{-1,8}
$$

Sans attribuer à cette relation la valeur d'une $10 i$ de propagation (elle peut représenter l'enveloppe de plusieurs lois différentes, par exemple si un terme exponentiel devait être ajouté) elle est vérifiée par plus de 600 mesures effectuées sur divers chantiers, et elle est utilisée couramment pour juger de la nécessité de procéder à des contrôles et pour procéder au réglage des appareils avant les mesures.

\section{CONCLUSIONS}

L'analyse des mesures effectuées sur des chantiers de terrassements à l'explosif a permis de mettre en évidence une relation simple entre vitesse de vibration maximum, charge et distance. Si cette relation ne représente pas un modèle théorique rigoureux, elle peut se révèler utile pour la prévision du niveau maximum des vibrations produites par les tirs d'explosifs tels qu'ils sont couramment pratiqués en travaux publics.

\section{BIBL,IOGRAPHTE}

NICHOLIS H.R., JOHNSON C.F., DUVALI W.I., 1971 Blasting vibrations and their effects on structures. U.S. Bureau of mines - bulletin 656 .

WUSTENHAGEN K. 1973 - Felsdynamische Untersuchungsmethoden in der Baupraxis - Rock Mechanics.

HENDRON A.J., DOWDING C.H., 1974, - Ground and structural response due to blasting.

C.R. du 3ème Congrès de la Société Internationale de Mécanique des Roches, DENNER.

CHAPOT P., - Etude des vibrations provoquées par les explosifs dans les massifs rocheux. Rapport de recherche des Laboratoire des Ponts et Chaussées à paraiture. 